\title{
The Influence of Operative Approach on Food Retention after Open and Laparoscopy-Assisted Distal Gastrectomy (LADG) for Gastric Cancer
}

\author{
Chang Yul Kang, M.D., Hong Rae Cho, M.D., Gyu Yeol Kim, M.D. \\ Department of Surgery, Ulsan University Hospital, University of Ulsan College of Medicine, Ulsan, Korea
}

Purpose: Laparoscopy-assisted distal gastrectomy (LADG) has gained wide acceptance for minimally invasive treatment of early gastric cancer (EGC). The aim of this study was to see the relationship between the operative approach of a distal subtotal gastrectomy and food retention of the remnant stomach.

Methods: A retrospective review of the records of 321 consecutive patients with gastric cancer who underwent a dista subtotal gastrectomy between 2001 and 2008 was conducted. A total of 233 patients who revisited the same surgeon's outpatient clinic and received regular endoscopic examination using the same protocol were finally included in this study. Reconstruction was performed using the Billroth I procedure. Mechanical-stapled anastomosis (MSA) was performed in 112 patients and conventional hand-sutured anastomosis (HA) was performed in 121 patients.

Results: According to results of multivariate analysis, the anastomosis method (MSA) was the only independent risk fac- tor for accumulation of food residue. At six and 12 months after surgery, the incidence of food retention was higher in patients who had undergone MSA $(22.3 \%, 13.4 \%)$ than in those who had undergone HA $(9.1 \%, 2.5 \%)(p=0.006$, $p=0.002$, respectively). However, the incidence of food residue at 24 months after surgery did not differ statistically between MSA and HA $(p=0.266)$.

Conclusion: Our results showed that the laparoscopic approach was not influenced on the accumulation of food residue. Mechanical-stapled anastomosis was the only independent risk factor for food retention. During the early postoperative period, although more food retention was observed in patients who underwent MSA than in those who underwent $\mathrm{HA}$, in the long term, this anastomosis method did not influence food retention after a distal gastrectomy.

Key words: Gastric cancer, Operative approach, Food retention, Anastomosis method (MSA, HA)

\section{INTRODUCTION}

Gastric cancer is still a major health problem and leading cause of cancer death in spite of decreasing worldwide incidence. Especially, Korea and Japan have the highest prevalence of gastric cancer in the world. ${ }^{1}$ The proportion of early gastric cancer (EGC) to advanced gastric cancer is increasing with the development of mass screening methods. Owing to early detection of the disease, the results of treatment for gastric cancer have improved in Korea. ${ }^{2}$ Considering the excellent prognosis of EGC, the quality of life after operation has been focused on these patients. For the better quality of life, laparoscopic gastrectomy has emerged as an alternative treatment option for EGC patients. ${ }^{3}$ The purposes of

Received September 28, 2012, Revised 1st, October 18, 2012; 2nd, October 19, 2012, Accepted October 19, 2012

※ Corresponding author : Gyu Yeol Kim

Department of Surgery, Ulsan University Hospital, University of Ulsan College of Medicine, 290-3, Jeonha-dong, Dong-gu, Ulsan 682-714, Korea

Tel : +82-52-250-7109, Fax : +82-52-250-8071

E-mail : kim9107798@dreamwiz.com

http://dx.doi.org/10.7602/jmis.2012.15.4.114 laparoscopic surgery for gastric cancer are to minimize surgical insults and to maximize patient's quality of life, while not influencing radicality. A number of reports have presented the excellent short term outcomes; less postoperative pain, improved cosmetics, less inflammatory reaction, a good preserved immune function, a rapid recovery of bowel function, shorter hospital stay, and a rapid return to normal social activity. ${ }^{4-6}$

The frequency of recurrent cancer in the gastric remnant after distal gastrectomy is around $1 \sim 5 \%$ and it is known that $20 \%$ of the patients who died from the recurrence of gastric cancer had recurrent cancer that was limited to the gastric remnant. ${ }^{9}$ It is recently reported that the incidence of gastric cancer arising in the remnant stomach is increasing owing to prolonged survival of gastric cancer patients. ${ }^{10}$ The risk of recurrent cancer in the gastric remnant is known to be proportional to the amount of time after the operation. Early recurrence may be caused by microcarcinomas in the gastric remnant because the frequency of synchronous gastric cancer is as high as $6 \sim$ $14 \%{ }^{11-13}$ Early detection and appropriate management for remnant gastric cancer can result in a favorable outcome., ${ }^{7,10,14}$ Thus, the surveillance endoscopy has become important for follow-up programs for patients that have undergone a subtotal gastrectomy, when viewed from the standpoint of cancer detection 
in the remnant stomach as well as for functional evaluation., ${ }^{7,10}$ However, food retention has been frequently observed during surveillance endoscopic examinations, and has occasionally hindered the complete observation of the remnant stomach. Also the long term quality of life of laparoscopic surgery for gastric cancer is not clearly revealed especially regards to the food retention.

We have been performed LADG for EGC since 2006 in our hospital. The food residue in the remnant stomach has been observed increasingly in the patients those who have received follow-up endoscopic examination after LADG. Occasionally the complete observation of remnant stomach was hindered due to the food residue. Even there were some of patients who received the endoscopic examination $1 \sim 2$ days after they hospitalized with fast. So, we assumed that the operative approach for gastric cancer influenced on the incidence or degree of food retention. Nevertheless, few investigations have documented the incidence or significance of food retention after LADG. We analyzed the relationship between the incidence of food retention of the remnant stomach and the operative and clinical factors. Especially, we aimed to elucidate the relationship between the operative approach of a distal gastrectomy and the food retention of the remnant stomach.

\section{MATERIALS AND METHODS}

\section{1) Patients}

The records of 321 consecutive patients with gastric cancer that underwent a distal subtotal gastrectomy by one surgeon $(\mathrm{G}$. Y. Kim) at Department of Surgery, Ulsan University Hospital (UUH), University of Ulsan College of Medicine, Korea between April 2001 and December 2008 were reviewed retrospectively. Eighty eight patients were excluded from the study for the following reasons: Seventy four patients did not receive endoscopic examination regularly during the follow-up periods. Fourteen patients were performed Billroth II reconstruction. One patient was performed balloon dilatation for postoperative stenosis of anastomotic site. A total of 233 patients who revisited the outpatient clinic and received endoscopic examination regularly were finally included in this study. Initially, we included only $\mathrm{T} 1$ and $\mathrm{T} 2$ gastric cancer because of elimination of bias of affect to function of remnant stomach by chemotherapy for advanced gastric cancer. In all included patients, reconstruction was performed using the Billroth I procedure. Mechanical-stapled anastomosis (MSA) was done in 112 patients and conventional hand-sutured anastomosis (HA) was done in 121.

\section{2) Operative approach}

LADG consisted of the following procedures ${ }^{15,16}$ : (1) laparoscopic dissection of lesser omentum and greater omentum, mobilization of the stomach, ligation and division of right gastroepiploic vessels, right gastric vessels, and left gastric vessels under pneumoperitoneum and (2) resection of the distal two thirds of the stomach followed by MSA or HA between the gastric remnant and duodenal stump through a $5 \mathrm{~cm}-$ long epigastric mini-laparotomy incision by extracorporeal manner. Conventional open distal gastrectomy was performed in a usual manner through an upper midline laparotomy incision from xiphoid process to umbilicus.

\section{3) Anastomosis method}

Hand suturing was done by Albert-Lembert anastomoses. Albert anastomoses were performed with 2-0 Vicryl (Ethicon; Johnson \& Johnson, Somerville, NJ, USA) running sutures. Lembert anastomoses were done with 3-0 silk knotted sutures. Mechanical-stapled anastomosis was performed using PCEEA-28 mm staplers (United States Surgical, Norwalk, CT, USA). The procedure has been described previously by Nance. ${ }^{17}$ The anvil head was inserted into the cut end of the duodenum. The head of the circular stapler was introduced into the stomach through the gastrotomy, $5 \mathrm{~cm}$ from the gastric closure. Then, the anastomosis was performed on the greater curvature of the gastric closure. Gastroduodenostomy was performed in a manner similar to that used with the hand suturing technique.

\section{4) Postoperative UGI endoscopic examination}

All patients revisited the outpatient clinic, and were examined according to the Ulsan Universitiy Hospital (UUH) follow-up protocol, which included clinical examination and laboratory study. These patients had undertaken a follow-up endoscopic examination at every 6 months after the surgery at gastrointestinal endoscopy center in UUH. All patients received endoscopic examination regularly during the follow-up periods. The patients were given written instructions about diet preparation before the examinations. Diet preparation for the endoscopic examinations included a soft meal diet at 6 p.m. in the evening the day before the endoscopy and fasting from 8:00 p.m. until the endoscopic examination. All endoscopic examinations were performed between 9:00 a.m. and 12:00 p.m.

\section{5) Degree of food residue}

Food retention was defined as the presence of liquid or solid food material in the remnant stomach. Kubo et al. ${ }^{18}$ proposed 
the amount of residual food was classified into five grades, as follows: grade 0 , no residual food; grade 1, a small amount of residual food; grade 2, a moderate amount of residual food, but possible to observe the entire surface of the remnant stomach with body rolling; grade 3 , a moderate amount of residual food, which hinders observation of the entire surface even with body rolling; and grade 4, a great amount of residual food, for which endoscopic observation is impossible. We determined positive for food residue when the grade is 3 or above in our hospital. The assessment was confirmed by reviewing endoscopy records or endoscopic photographs. Stenosis and obstruction of anastomotic site were excluded from the cases because our issue was functional access rather than structural derangement. All of the patients found to have food retention by the endoscopic examination received a dietary education.

\section{6) Statistics}

SPSS 19.0 (IBM SPSS Inc., Chicago, IL) for statistical anal- ysis was employed for $\chi^{2}$ test and binary logistic regression to analyze the data. The level of signficance was set at $p<0.05$.

\section{RESULTS}

\section{1) Food residue and clinicopathological and operation-related factors}

We analyzed the relationship between food residue and clinicopathological and operation-related factors at postoperative 6 moths. At postoperative 6 moths, overall incidence of food retention was $15.5 \%$ (36 of the 233 examinations). The incidence of food retention in patients who had performed LADG $(20.9 \%)$ was higher than ODG group $(10.9 \%) \quad(p=0.045)$.

\section{2) Univariate analysis}

The results of univariate analysis of the relationship between clinicopathological factors and food-residue-negative and -positive patients was shown at Table 1 . All of clinical factors

Table 1. Relationship between clinicopathological factors and food residue

\begin{tabular}{|c|c|c|c|}
\hline & \multicolumn{3}{|c|}{ No. of patients (\%) } \\
\hline & Food residue negative $(\mathrm{n}=197)$ & Food residue positive $(n=36)$ & $p$ value* \\
\hline Age (years) & & & 0.465 \\
\hline$\geq 60$ & $83(42.1)$ & $18(50.0)$ & \\
\hline$<60$ & $114(57.9)$ & $18(50.0)$ & \\
\hline Gender & & & 0.851 \\
\hline Male & $126(64.0)$ & $22(61.1)$ & \\
\hline Female & $71(36.0)$ & $14(38.9)$ & \\
\hline Body mass index $\left(\mathrm{kg} / \mathrm{m}^{2}\right)$ & & & 0.451 \\
\hline$<25$ & $129(65.5)$ & $21(58.3)$ & \\
\hline$\geq 25$ & $68(34.5)$ & $15(41.7)$ & \\
\hline Underlying disease $^{\dagger}$ & $15(7.6)$ & $7(19.4)$ & 0.350 \\
\hline History of abdominal operation & $16(8.1)$ & $5(13.9)$ & 0.337 \\
\hline AJCC stage ${ }^{\ddagger}$ & & & 0.768 \\
\hline IA & $160(81.2)$ & $31(86.1)$ & \\
\hline IB & $20(10.2)$ & $2(5.6)$ & \\
\hline IIA & $14(7.1)$ & $2(5.6)$ & \\
\hline IIB & $3(1.5)$ & $1(2.8)$ & \\
\hline Histologic type & & & 0.660 \\
\hline \multicolumn{4}{|l|}{ Adenocarcinoma } \\
\hline WD & $36(18.3)$ & $4(11.1)$ & \\
\hline MD & $53(26.9)$ & $11(30.6)$ & \\
\hline PD & $57(28.9)$ & $13(36.1)$ & \\
\hline SRC & $46(23.4)$ & $8(22.2)$ & \\
\hline MC & $5(2.5)$ & $0(0.0)$ & \\
\hline
\end{tabular}

${ }^{*}$ Cross-table analysis using $\chi^{2}$ test; ${ }^{\dagger}$ Underlying disease: Diabete Mellitus, thyroid disease, Rheumatoid arthritis; ${ }^{\dagger}$ Cancer staging according to the American Joint Committee on Cancer $\left(7^{\text {th }}\right.$ ed., 2010). WD $=$ well differentiated; $\mathrm{MD}=$ moderate differentiatd; $\mathrm{PD}=$ poor differentiated; $\mathrm{SRC}=$ signet ring cell carcinoma; $\mathrm{MC}=$ mucinous carcinoma. 
including underlying disease, age, distribution of gender, preoperative body mass index (BMI) and history of abdominal operation were no significant difference between food-residue-negative and -positive patients. Also, the histologic types and pathologic TNM stages were similar to food-residue-negative and -positive patients. Table 2 show the relationships between operation-related factors and food-residue-negative and -positive patients. Higher incidence of food retention was found in patients who had undergone laparoscopy-assisted distal gastrectomy (LADG) than open distal gastrectomy (ODG). And, we found more frequent food residue accumulation in patients who had performed the MSA than HA.

\section{3) Multivariate analysis}

In multivariate logistic regression analysis (Table 3), the presence or absence of food residue served as the outcome variable, and operative approach (LADG vs ODG) and anastomosis method (MSA vs HA) served as modifier variables. Multivariate analysis shows that anastomosis method (MSA) was only one independent risk factor for a positive finding of food residue.

\section{4) Chronological incidence of food retention}

The overall incidence of food retention was $15.5 \%$ at 6 months, $7.7 \%$ at 12 months, and $3.0 \%$ at 24 months after distal subtotal gastrectomy. The chronological observations for the incidence of food retention are shown in Fig. 1. The incidence of food residue was higher in patients who had performed MSA $(22.3 \%, 13.4 \%)$ than HA $(9.1 \%, 2.5 \%)$ at 6 and 12 months after

Table 2. Relationship between operation-related factors and food residue

\begin{tabular}{lrcc}
\hline & \multicolumn{3}{c}{ No. of patients (\%) } \\
\cline { 2 - 4 } & $\begin{array}{c}\text { Food residue } \\
\text { negative } \\
(\mathrm{n}=197)\end{array}$ & $\begin{array}{c}\text { Food residue } \\
\text { positive } \\
(\mathrm{n}=36)\end{array}$ & $\begin{array}{c}p \\
\text { value* }\end{array}$ \\
& $49(24.9)$ & $5(13.9)$ & \\
\hline $\begin{array}{l}\text { Operating time (min) } \\
\quad<180\end{array}$ & $148(75.1)$ & $31(86.1)$ & \\
$\quad \begin{array}{l}\text { 180 } \\
\text { Operative approach }\end{array}$ & $83(42.1)$ & $22(61.1)$ & \\
$\quad$ LADG & $114(57.9)$ & $14(38.9)$ & \\
$\quad$ ODG & $87(44.2)$ & $25(69.4)$ & $\mathbf{0 . 0 0 6}$ \\
Anastomosis method & $110(55.8)$ & $11(30.6)$ & \\
$\quad \begin{array}{l}\text { MSA } \\
\text { HA }\end{array}$ &
\end{tabular}

LADG = laparoscopy-assisted distal gastrectomy; $\quad$ ODG $=$ open distal gastrectomy; MSA = mechanical-stapled anastomosis; HA= hand-sutured anastomosis. ${ }^{*}$ Cross-table analysis using $\chi^{2}$ test. the surgery ( $p=0.006, p=0.002$, respectively). At 24 months after the surgery, the incidence of food retention revealed no statistical difference between MSA and HA $(p=0.266)$.

\section{5) Food retention in laparoscopic era}

We reviewed the all patients' clinicopathological and operation-related factors according to operative approaches. The clinical factors including age, distribution of gender, preoperative body mass index (BMI), underlying disease and history of abdominal operation were no significant difference between patients who performed LADG and ODG. TNM staging and histologic type were similar to patients who performed LADG and ODG. Patients on underwent LADG performed MSA more than HA $(83.0 \%$ vs $9.9 \%, p<0.001)$ (Table 4$)$. We

Table 3. Multivariate analysis of risk factors associated with food residue

\begin{tabular}{lccc}
\hline & \multicolumn{3}{c}{ No. of patients (\%) } \\
\cline { 2 - 4 } & $\begin{array}{c}\text { Relative } \\
\text { risk }\end{array}$ & $\begin{array}{c}95 \% \text { confidence } \\
\text { interval }\end{array}$ & $\begin{array}{c}p \\
\text { value* }\end{array}$ \\
\hline Age $(\geq 60$ years) & 1.373 & $0.648 \sim 2.913$ & 0.408 \\
Gender & 1.181 & $0.560 \sim 2.489$ & 0.662 \\
Operative approach (LADG) & 1.181 & $0.408 \sim 3.417$ & 0.759 \\
Anastomosis method (MSA) & 2.874 & $1.340 \sim 6.162$ & $\mathbf{0 . 0 0 7}$ \\
\hline
\end{tabular}

LADG = laparoscopy-assisted distal gastrectomy; MSA = mechanicalstapled anastomosis. *Multivariate analysis using binary logistic regression.

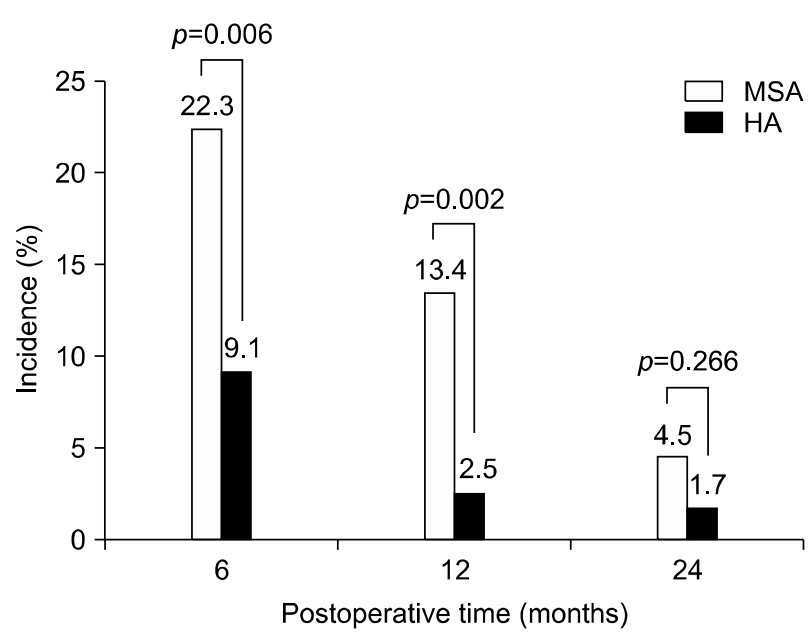

Fig. 1. The graph shows the incidence of food residue according to the anastomosis method during the postoperative periods. MSA = mechanical-stapled anastomosis; $\mathrm{HA}=$ hand-sutured anastomosis. 
Table 4. Relationship between operation-related factors and anastomosis method

\begin{tabular}{lccc}
\hline & \multicolumn{3}{c}{ No. of patients (\%) } \\
\cline { 2 - 4 } & MSA $(\mathrm{n}=112)$ & HA $(\mathrm{n}=121)$ & $p$ value* $^{*}$ \\
\hline Operative approach & & & $<\mathbf{0 . 0 0 1}$ \\
LADG & $93(83.0)$ & $12(9.9)$ & \\
ODG & $19(17.0)$ & $109(90.1)$ & \\
\hline
\end{tabular}

LADG = laparoscopy-assisted distal gastrectomy; $\mathrm{ODG}=$ open distal gastrectomy; MSA = mechanical-stapled anastomosis; HA $=$ hand-sutured anastomosis. ${ }^{*}$ Cross-table analysis using $\chi^{2}$ test.

also reviewed the affect by the learning curve of LADG. Until 60th cases of LADG, there were 11 cases $(50.0 \%)$ of food retention at 6 months after the surgery. Among the remaining 45 cases of LADG, there were $11(50.0 \%)$ cases thereafter (Table $5)$.

\section{DISCUSSION}

We were interested in the food residue, which was more frequently observed in the postoperative follow-up endoscopic examinations after performing LADG, and which might be relevant to the quality of life after gastric surgery. Also, food retention has occasionally hindered the complete observation of the remnant stomach. We attempted to reveal whether food retention is the limitation of laparoscopic surgery or not. We selected only patients who were follow up by one surgeon in our outpatient clinic examined according to the same follow-up protocol because of elimination of technical bias of multiple operator.

Kubo et al. ${ }^{18}$ proposed an endoscopic classification assessing several aspects of the remnant stomach, and compared the endoscopic grade of residual food in the remnant stomach after three types of reconstruction methods (Roux-en-Y, Billroth I, and a pylorus preserving gastrectomy). Their classification (RGB classification: Residue, Gastritis, Bile) can be used easily and is practical. Despite the interval between the distal gastrectomy and endoscopic examination was not addressed, the incidence of residual food was $14.0 \%$ in the Roux-en-Y group, $22.3 \%$ in the Billroth I group and $37.5 \%$ in the pylorus preserving gastrectomy group. ${ }^{13,14}$ This classification seems to be useful to describe these findings and to further evaluate these reconstructive methods.

The relationships between the reconstruction procedure after distal gastrectomy and food retention have been studied. Jung
Table 5. Relationship between learnig curve and food residue in LADG GD

\begin{tabular}{cccc}
\hline & \multicolumn{3}{c}{ No. of patients (\%) } \\
\cline { 2 - 4 } & $\begin{array}{c}\text { Food residue } \\
\text { negative }(\mathrm{n}=83)\end{array}$ & $\begin{array}{c}\text { Food residue } \\
\text { positive }(\mathrm{n}=22)\end{array}$ & $p$ value* \\
\hline $\begin{array}{c}\text { Learning curve } \\
\leq 60^{\text {th }} \text { case } \\
>60^{\text {th }} \text { case }\end{array}$ & $49(59.0)$ & $11(50.0)$ & 0.476 \\
\end{tabular}

LADG = laparoscopy-assisted distal gastrectomy; GD = gastroduodenostomy. ${ }^{*}$ Cross-table analysis using $\chi^{2}$ test.

et al. ${ }^{19}$ had divided the amount of residual food into four grades, which were modified by the endoscopists in National Cancer Center by referring to the classification system reported by Kubo et al. ${ }^{18}$ They had compared the Billroth I with Billroth II procedures in terms of food retention and time interval. The food residue score was higher in Billroth I group of patients than Billroth II group at 3 months after surgery (mean score 1.49 vs 1.04: $p=0.006$ ). The food residue scores at 12 and 24 months after surgery revealed no statistical difference between the two groups $(p=0.076, p=0.684$, respectively). In present study, there was no statistical significance in reconstruction method and food residue (data not shown). The reason that we are assuming is due to very low number of the Billroth II than the Billroth I. Thus, patients who performed Billroth II (14 patients; MSA 13 patients, HS 1 patient) were excluded from this study.

Other numerous factors have been associated with food retention after a gastrectomy. Watanabe et al. ${ }^{20}$ reported that underlying endocrine or metabolic diseases (thyroid disease, diabetes mellitus, SLE), postoperative gastric retention, and Billroth I reconstruction were independent risk factors for food residue in a multivariate analysis. Postoperative gastric retention was occasionally observed 7,10 , or more days after gastric surgery. The causes of these complications were luminal obstruction at the stoma and gastric atony resulting from vagal denervation. Diet preparation significantly decreased the incidence of food residue. In this study, there was no statistical significance between underlying disease and food retention. Regards to the underlying diseases in this study, there were three diabetes mellitus, two thyroid disease, two diabetes mellitus combination with thyroid disease in case of Food-residue-positive examination $(n=36)$ at 6 months after surgery (Table 1).

In 1979, the first MSA technique using a circular stapler after 
distal gastrectomy was reported by Nance. ${ }^{17}$ Hori et al. ${ }^{21}$ reported the results of a prospective study comparing the clinical outcome after MSA with that after HA for B-I reconstruction. Although the total operation time did not differ significantly between the groups, the time required for anastomosis was significantly shorter in the stapled group $(14 \pm 5.9 \mathrm{~min})$ than in the hand-sutured group $(25 \pm 6.9 \mathrm{~min})(p=0.02)$. Their study was a valuable prospective randomized trial, but the remnant stomach was not evaluated after surgery.

Takahashi et al. $^{22}$ reported that The operative time for B-I reconstruction with distal gastrectomy was significantly shorter with MSA than with HA. While there were no significant disadvantages in the incidence of complications associated with MSA compared with HA, MSA resulted in more residue in the remnant stomach. They analyzed upper gastrointestinal endoscopic findings 1 year after surgery, and classified the status of the remnant stomach using the Residue, Gastritis, Bile (RGB) classiffication system reported by Kubo et al. ${ }^{18}$ In the mechanical-stapled anastomosis (MSA) group, 37 (69\%) of 54 patients had residual food in the remnant stomach, whereas in the hand-sutured anastomosis (HA) group, only 15 (31\%) of 49 patients had residual food $(p<0.01) .^{22}$

However, there have been few reports that have delineated the effect of the anastomosis method and operative approach and the incidence of food retention after distal subtotal gastrectomy for gastric cancer. As far as we know, this is the first study that has compared the LADG with OGD in terms of food retention and time interval. In this study, a significantly higher rate of food residue accumulation was found in patients who had undergone laparoscopic assisted distal gastrectomy (LADG). But, multivariate analysis shows that anastomosis method (MSA) was only one independent risk factor for a positive finding of food residue. This was because the patients who underwent LADG also had performed MSA much more compare to the patients those who underwent ODG.

Kim et al. ${ }^{23,24}$ had reported that the learning curve of LADG has been demonstrated that the number of cases required to achieve competence in LADG is 30th to 60th cases. We tried to reveal the unexperienced technique caused the food retention of remnant stomach after gastrectomy. In present study, until 60 th cases of LADG, there were 11 cases $(50.0 \%)$ of food retention at 6 months after the surgery. Among the remaining 45 cases of LADG, there were $11(50.0 \%)$ cases thereafter (Table $5)$. Thus, the frequently found food retention in laparoscopic approach surgery is not the LADG learning curve related bias.

In this study, interestingly, the incidence of food residue was likely to decrease until 2 years after distal subtotal gastrectomy
(Fig. 1). The frequency of food retention was higher in MSA than HA but decreased by about $20 \%$ at 2 years after surgery. A possible explanation for this change may be that the patients who had food retention received dietary education in the outpatient clinic and as time goes by, perhaps the remnant stomach adapted to recover gastrointestinal motility after the surgery. We claim that dietary modification, with patients being advised to eat frequent, small meals rather than large meals, is important in reducing the degree of food residue. Yet, the statistics show that the food retention rate at 6 and 12 months after surgery was significantly higher in MSA than HA. Takahashi et al. $^{22}$ assumed that food stasis associated with MSA may be caused by several factors, including gastric motility and the location of the anastomosis creating a sharp angle between the remnant stomach and the duodenum. However, as far as I am concerned, there is nearly no such comparison study between anastomosis method and food retention. Further studies are required for the better understanding of the reasons for this accumulation of residual food with MSA and to provide a solution to this problem.

The clinical significance of food retention is controversial. Watanabe et al. ${ }^{20}$ reported that the patients with food residue did not complain of any special symptoms, did not receive any treatment to remove it, and did not need any nutritional support. Therefore, they considered that the clinical significance of food residue in the gastric remnant is that it constitutes an obstacle to intragastric observation and a risk factor for respiratory aspiration of stomach contents during endoscopy. Also, in this study, patients with food residue did not complaint any special symptom. However, a hindrance to the complete endoscopic evaluation of the remnant stomach alone may have clinical significance, because the surveillance endoscopy is crucial follow-up program for early detection and appropriate management for remnant gastric cancer.

\section{CONCLUSION}

We found more frequently food retention of remnant stomach after laparoscopic distal gastrectomy than open approach. But, the operative approach was not influenced on the accumulation of food residue. Anastomosis method (MSA) was only independent risk factors for a positive finding of food residue. More food residue was detected in patients underwent MSA than HA, but we suggest that the presence of food residue as seen on a follow-up endoscopic examination may have no clinical implications in 2 years after gastrectomy. The limitation of this study is retrospective, so further prospective studies are 
required to better understanding of the reason for this accumulation of food residue.

\section{REFERENCES}

1) Crew KD, Neugut AI. Epidemiology of gastric cancer. World J Gastroenterol 2006;12:354-362.

2) Ahn HS, Lee HJ, Yoo MW, et al. Changes in clinicopathological features and survival after gastrectomy for gastric cancer over a 20-year period. Br J Surg 2011;98:255-260.

3) Kim HH, Ahn SH. The current status and future perspectives of laparoscopic surgery for gastric cancer. J Korean Surg Soc 2011;81:151-162.

4) Kim HH, Hyung WJ, Cho GS, et al. Morbidity and mortality of laparoscopic gastrectomy versus open gastrectomy for gastric cancer: an interim report--a phase III multicenter, prospective, randomized Trial (KLASS Trial). Ann Surg 2010; 251:417-420.

5) Huscher CG, Mingoli A, Sgarzini G, et al. Laparoscopic versus open subtotal gastrectomy for distal gastric cancer: five-year results of a randomized prospective trial. Ann Surg 2005;241:232-237.

6) Kim YW, Baik YH, Yun YH, et al. Improved quality of life outcomes after laparoscopy-assisted distal gastrectomy for early gastric cancer: results of a prospective randomized clinical trial. Ann Surg 2008;248:721-727.

7) Thorban S, Bottcher K, Etter M, Roder JD, Busch R, Siewert JR. Prognostic factors in gastric stump carcinoma. Ann Surg 2000;231:188-194.

8) Ohashi M, Katai H, Fukagawa T, Gotoda T, Sano T, Sasako M. Cancer of the gastric stump following distal gastrectomy for cancer. Br J Surg 2007;94:92-95.

9) Huguier M, Ferro L, Barrier A. Early gastric carcinoma: spread and multicentricity. Gastric Cancer 2002;5:125-128.

10) Takeno S, Noguchi $T$, Kimura $Y$, Fujiwara $S$, Kubo $N$, Kawahara K. Early and late gastric cancer arising in the remnant stomach after distal gastrectomy. Eur J Surg Oncol 2006;32:1191-1194.

11) Bearzi I, Ranaldi R. Multifocal early gastric cancer: morphology and histogenesis. Pathol Res Pract 1986;181:144-147.

12) Kosaka T, Miwa K, Yonemura $Y$, et al. A clinicopathologic study on multiple gastric cancers with special reference to distal gastrectomy. Cancer 1990;65:2602-2605.

13) Otsuji E, Kuriu Y, Ichikawa D, Okamoto K, Hagiwara A, Yamagishi $\mathrm{H}$. Clinicopathologic characteristics and prognosis of synchronous multifocal gastric carcinomas. Am J Surg 2005;189:116-119.

14) Inomata M, Shiraishi N, Adachi $Y$, Yasuda K, Aramaki M, Kitano S. Gastric remnant cancer compared with primary proximal gastric cancer. Hepatogastroenterology 2003;50:587-591.

15) Kitano S, Iso Y, Moriyama M, Sugimachi K. Laparoscopy-assisted Billroth I gastrectomy. Surg Laparosc Endosc 1994;4:146-148.

16) Shiraishi N, Adachi $Y$, Kitano $S$, Bandoh $T$, Katsuta $T$, Morimoto A. Indication for and outcome of laparoscopyassisted Billroth I gastrectomy. Br J Surg 1999;86:541-544.

17) Nance FC. New techniques of gastrointestinal anastomoses with the EEA stapler. Ann Surg 1979;189:587-600.

18) Kubo M, Sasako M, Gotoda $T$, et al. Endoscopicevaluation of the remnant stomach after gastrectomy: Proposal for a new classification. Gastric Cancer 2002;5:83-89.

19) Jung HJ, Lee JH, Ryu KW, et al. The influence of reconstruction methods on food retention phenomenon in the remnant stomach after a subtotal gastrectomy. J Surg Oncol 2008;98:11-14.

20) Watanabe H, Adachi W, Koide N, Yazawa I. Food residue at endoscopy in patients who have previously undergone distal gastrectomy: Risk factors and patient preparation. Endoscopy 2003;35:397-401.

21) Hori S, Ochiai S, Gunji Y, Hayashi H, Suzuki T. A prospective randomized trial of hand-sutured versus mechanically stapled anastomoses for gastroduodenostomy after distal gastrectomy. Gastric Cancer 2004;7:24-30.

22) Takahashi T, Saikawa Y, Yoshida M, et al. Mechanical-stapled versus hand-sutured anastomoses in Billroth-I reconstruction with distal gastrectomy. Surg Today 2007;37:122-126.

23) Kim MC, Jung GJ, Kim HH. Learning curve of laparoscopyassisted distal gastrectomy with systemic lymphadenectomy for early gastric cancer. World J Gastroenterol 2005;11:7508-7511.

24) Kim JH, Jung YS, Kim BS, et al. Learning curve of a laparoscopy assisted distal gastrectomy for a surgeon expert in performing a conventional open gastrectomy. J Korean Gastric Cancer Assoc 2006;6:167-172. 